\title{
Focalização do Pronaf na região Sul do Brasil
}

\author{
Focusing of Pronaf in Southern Brazil
}

\author{
Marcos Aurélio Brambilla e Ednaldo Michellon*
}

\begin{abstract}
Resumo: O objetivo do presente estudo foi avaliar a relação espacial do percentual de agricultores familiares e do financiamento do Programa Nacional de Fortalecimento da Agricultura Familiar (Pronaf) per capita por município na região Sul, que foi a região que recebeu aproximadamente 3 bilhões de reais em 2006, ou seja, mais de $40 \%$ dos recursos totais. Para isso, o trabalho utilizou como metodologia a Análise Exploratória dos Dados Espaciais (AEDE) e realizou as análises global (I de Moran) e local (mapa de cluster). Os dados foram extraídos do Ministério do Desenvolvimento Agrário (MDA), no ano de 2006. O resultado mostrou que há uma autocorrelação espacial positiva entre o percentual de agricultores familiares e o valor do financiamento do Pronaf per capita na região Sul do país. Concluiu-se que as mesorregiões Centro-Oriental e Sudeste do estado do Rio Grande do Sul apresentaram maior concentração de cluster do tipo alto-alto (AA). Por outro lado, as mesorregiões do Norte, Noroeste e Metropolitana de Curitiba no estado do Paraná e as mesorregiões do Vale do Itajaí, Norte e na Grande Florianópolis de Santa Catarina apresentaram as maiores concentrações de clusters do tipo baixo-baixo (BB).
\end{abstract}

Palavras-chave: Crédito rural; Análise exploratória de dados espaciais; Região Sul

\begin{abstract}
The objective of the present study was to evaluate the spatial relationship of the family farmers percentage and the financing of the National Program for Strengthening Family Agriculture (Pronaf) per capita by municipality in the South, the region that received approximately 3 billion reais in 2006, i.e., more than $40 \%$ of total resources. For such, the study used as Exploratory Analysis of Spatial Data (AEDE) as the methodology and performed the global (I de Moran) and local (cluster map) analyses. Data were retrieved from the Ministry of Agrarian Development (MDA) in 2006. The result showed that there is a positive spatial autocorrelation between the percentage of family farmers and the value of the financing of Pronaf per capita in the southern region of the country. It was concluded that the central-eastern and southeastern mesoregions of the state of Rio Grande do Sul presented a higher concentration of the high-high cluster (AA). On the other hand, the mesoregions of the North, Northwest and the Metropolitan of Curitiba in the state of Paraná and the mesoregions of the Vale do Itajaí, Norte and in the Great Florianópolis of Santa Catarina presented the highest concentrations of clusters of the low-low type (BB).
\end{abstract}

Keywords: Rural credit; Exploratory spatial data analysis; Southern region

JEL Code: J18

\footnotetext{
* Respectivamente, Professor dos cursos de Administração e Ciências Contábeis do Instituto Adventista Paranaense (IAP). E-mail: marcos-brambilla@hotmail.com; e Professor do Programa de Pós-Graduação em Ciências Econômicas da Universidade Estadual de Maringá (UEM). E-mail: emichellon@uem.br. DOI: http://dx.doi.org/10.5380/re.v40i72.55474
} 


\section{Introdução}

Até o início dos anos 1990, não havia política pública na esfera nacional destinada especificamente aos agricultores familiares. Entretanto, a Constituição de 1988 sugeriu que as ações dos estados fossem mais descentralizadas, e isso permitiu alterações nas políticas sociais de distribuição de recursos públicos. Nesse contexto, e, com as pressões dos movimentos sociais, surgiu o Programa Nacional de Fortalecimento da Agricultura Familiar (Pronaf) em 1995/96 (MATTEI, 2005).

O Pronaf é um programa destinado aos agricultores familiares e aos assentados da reforma agrária, no qual recebem crédito do programa para o financiamento de seus projetos. Dentre os financiamentos do meio rural, o programa tem as menores taxas e apresenta baixa inadimplência no mercado de crédito. $\mathrm{O}$ agricultor familiar, para obter um financiamento, deve, primeiramente, especificar qual a utilização desse financiamento, o qual pode ser remetido para o investimento em máquinas, equipamentos ou infraestrutura de produção e serviços agropecuários ou para financiar a safra ou atividade agroindustrial (MDA, 2016).

Assim, o programa tem o intuito gerar renda para os agricultores familiares e, com isso, também desenvolver a economia local, principalmente onde mais se concentram esses agricultores. De acordo com o Censo Agropecuário de 2006, a região do país que obteve o maior repasse de recursos do Pronaf em 2006 é a região Sul, com 41\% do valor repassado; a segunda com maior valor é a região Nordeste, com 26\%; a terceira com o maior valor é a região Sudeste, com 19\% do valor; a quarta com o maior valor é a região Norte, com $8 \%$; e a região que obteve o menor repasse foi o Centro-Oeste, com 6\% do valor total repassado (BACEN, 2016). Desse modo, é possível observar que a região Sul recebeu mais recursos do Pronaf do que todas as demais regiões e, diante disso, este estudo foi realizado com o objetivo de identificar se, na região Sul, municípios com grande percentual de agricultores familiares estão recebendo mais recursos do Pronaf.

Portanto, surge a seguinte indagação: os recursos do Pronaf estão concentrados em regiões onde se concentra o maior número de agricultores familiares na região Sul? Para alcançar o objetivo descrito acima, o trabalho foi dividido em seis partes além desta introdução. A segunda parte discute as questões relacionadas a agricultura familiar e o Pronaf. A terceira parte aborda o Pronaf especificamente na região Sul. A quarta parte é referente a metodologia, o qual apresenta o método e as variáveis utilizadas. A quinta parte apresenta os resultados 
e as discussões do trabalho. E a sexta, e última parte, exibe as considerações finais, seguida das referências.

\section{Agricultura familiar e o Pronaf}

A agricultura familiar possui algumas peculiaridades em relação à agricultura não familiar, sendo as principais o fato de a administração daquela ser realizada pela própria família e a produção agropecuária é sua principal fonte de renda.

Antes da década de 1990, não havia no Brasil políticas públicas voltadas aos agricultores familiares, sendo que essa classe ainda não tinha um conceito formal, que hoje é conhecido como agricultura familiar. Um dos fatores que contribuiu para esse aparecimento foram as crescentes reivindicações dos trabalhadores rurais a partir da Constituição de 1988. Além disso, os estudos realizados pela Organização das Nações Unidas para a Alimentação e a Agricultura (FAO), em parceria com o Instituto Nacional de Colonização e Reforma Agrária (INCRA), estabeleceram definições e critérios para a agricultura familiar com o objetivo de criar políticas públicas que beneficiassem os agricultores familiares (MATTEI, 2001).

Ademais, o Brasil, na década de 1990, foi marcado por representar um avanço do governo em incentivar a agricultura familiar. Em decorrência das mobilizações dos agricultores familiares, chamada de "Grito da Terra Brasil", em 1994, foi criado o Programa de Valorização da Pequena Produção Rural (PROVAP); o qual estabeleceu, pela Resolução 2101, de 24 de agosto de 1994, algumas normas para o crédito rural destinado aos pequenos agricultores rurais, tais como: apresentar $80 \%$ da renda proveniente da agricultura e não possuir vínculo empregatício permanente; realizar o repasse do crédito para grupos de até 20 agricultores, com contratações formais individuais; e o critério de uma área de até 4 módulos fiscais (MF) (BIANCHINI, 2015).

Em outras palavras, a década de 1990 é marcada pela mudança da estrutura do financiamento da atividade produtiva, e a política de crédito rural refletiu o contexto mais geral da redução do papel do governo na economia, buscando-se fomentar soluções de mercado, com a criação das instituições mais adequadas. $\mathrm{O}$ resultado final foi uma grande redução do volume de recursos disponíveis para o 
investimento, custeio e comercialização agrícola, especialmente a fonte de recursos do Tesouro, que reduziu 68\%, de $\mathrm{R} \$ 8.002$ milhões para $\mathrm{R} \$ 2.523$ milhões (MICHELLON; PELIZER, 2006).

Assim, em 1995, foi criado o Pronaf, programa que tem o objetivo de atender atividades agrícolas e não agrícolas dos agricultores familiares com repasses de custeio e investimento. Vale ressaltar que os recursos também são destinados a grupos coletivos e cooperativas de agricultores familiares (BATISTA; NEDER, 2014).

As condições iniciais para receber a Declaração de Aptidão (DAP) do Pronaf foram: explorar parte da terra na condição de arrendatário, posseiro, proprietário ou parceiro; não ter empregados permanentes, sendo que se houver a necessidade para atividade agrícola poderá receber ajuda de terceiros; não apresentar área superior a $4 \mathrm{MF}$; a sua renda total brutal anual ser de no mínimo $80 \%$ de atividades agrícola ou extrativa. O Pronaf, no decorrer dos anos, apresentou algumas modificações e uma grande evolução no número de contratos e volume de recursos (BIANCHINI, 2015).

É possível observar na Tabela 1 os contratos e o volume de recursos no período de 1999 a 2006. No ano de 1999, o número de contratos do Pronaf era de 791.677 e o financiamento era de $\mathrm{R} \$ 1.776 .951 .420,05$; em 2000, os contratos e o valor do financiamento aumentaram 5\%. Em 2001, os contratos caíram 4\% em relação ao ano anterior, porém o valor do financiamento subiu 19\%. E de 2001 a 2006, tanto os contratos como o volume de recursos do Pronaf aumentaram ano a ano, com um crescimento médio anual de $44 \%$ e $45 \%$, respectivamente. Entretanto, o montante de recursos por contrato de 2000 a 2004 apresentou um aumento médio de crescimento anual de 10\%; de 2004 para 2005, houve uma redução de 20\%; e, entre 2005 e 2006, os recursos médios por contrato voltaram a crescer $(7 \%)$.

$\mathrm{Na}$ literatura existem diversos estudos em que se analisam o crédito rural destinado à agricultura familiar por meio do Pronaf, como Mattei (2005), que procurou analisar o comportamento de indicadores de impactos do Pronaf em cem municípios que contrataram o maior volume de recursos do crédito rural disponibilizado no período de 2001-2004. Uma das conclusões foi que o crédito rural atende cada vez mais os agricultores familiares (MATTEI, 2005). 
Tabela 1 - Número de contratos e volume de recursos do Pronaf para o Brasil 1999-2006

\begin{tabular}{cccc}
\hline Ano & Número de contratos & Recursos (R\$) & $\begin{array}{c}\text { Média de recursos por contrato } \\
(\mathbf{R} \mathbf{)})\end{array}$ \\
\hline 1999 & 791.677 & $1.776 .951 .420,05$ & $2.244,54$ \\
2000 & 834.049 & $1.864 .888 .673,15$ & $2.235,95$ \\
2001 & 800.653 & $2.210 .744 .245,24$ & $2.761,17$ \\
2002 & 829.433 & $2.414 .869 .518,80$ & $2.911,47$ \\
2003 & 1.003 .837 & $3.158 .400 .036,64$ & $3.146,33$ \\
2004 & 1.345 .713 & $4.388 .790 .541,58$ & $3.261,31$ \\
2005 & 2.208 .198 & $5.785 .745 .810,94$ & $2.620,12$ \\
2006 & 2.551 .497 & $7.166 .030 .577,33$ & $2.808,56$ \\
\hline
\end{tabular}

Fonte: Elaboração própria a partir dos dados do Anuário estatístico do crédito rural - 2006.

Outro estudo realizado pelo mesmo autor, referente ao crédito rural, (Mattei, 2007) analisou o desempenho do Pronaf no Brasil, considerando sua concepção, principais instrumentos operacionais, objetivos e mudanças institucionais recentes do programa, em que analisa o programa em duas fases, primeiro até 1999 e depois até 2004. A principal conclusão é que o programa permanece concentrado na região Sul do país e, em termos dos grupos beneficiários, nota-se uma concentração dos recursos nos grupos $\mathrm{C}$ e $\mathrm{D}$, que são considerados os agricultores mais bem posicionados no sistema agropecuário familiar brasileiro.

O estudo de Pereira e Nascimento (2014) buscou avaliar a contribuição do Pronaf em reduzir o hiato entre a capacidade produtiva e o produto efetivo na agropecuária tocantinense. Para isso, foram utilizadas as regressões lineares pela média e quantílica. Os principais resultados mostraram que o setor agrícola tem grande relevância no estado do Tocantins, a grande participação da agricultura familiar na produção total agrícola e a forte presença do Pronaf, que além de contribuir com o reconhecimento da agriculta familiar, estimula a produção vegetal e animal nos municípios estudados.

Um estudo com intuito de analisar o efeito do Pronaf sobre a pobreza rural, realizado por Batista e Neder (2014), gerou estimativas de elasticidades Pronafrenda e Pronaf-desigualdade e utilizou o modelo de painel dinâmico. Os autores constataram que a elevação da renda per capita ou a redução da desigualdade de renda tendem a reduzir a pobreza; ii) o efeito do Pronaf sobre a renda per capita média e a desigualdade de renda está condicionado às especificidades 
socioeconômicas das unidades de observação; e iii) os gastos do Pronaf tendem a reduzir indiretamente a pobreza por meio da elevação da renda média e da redução da concentração de renda.

Também, Michellon, Kretzmann e Gonçalves (2007) analisaram a conjuntura do crédito rural comparando-se o montante destinado à agricultura familiar versus o destinado à agricultura patronal. Concluíram que o Pronaf contribuiu para elevar a participação do agronegócio familiar para $10,1 \%$ do Produto Interno Bruto brasileiro, contra 20,5\% do agronegócio patronal, pois o Pronaf impulsionou os grupos ligados à agricultura familiar para a luta em prol da ampliação do montante de crédito para o segmento, dado a participação no PIB do agronegócio ser, grosso modo, de 1 para 2 contra 1 para 5 do montante do crédito rural.

Outro trabalho realizado por Resende e Mafra (2016) procurou verificar, por meio de revisão de literatura e com dados oficiais sobre o Pronaf, como o programa é definido e avaliado, com o intuito de desconstruir a relação entre desenvolvimento e reconhecimento. Os principais resultados constataram que a distribuição de recursos e o modo como o programa é operacionalizado não são suficientes para atender os objetivos principais do Pronaf, que são reduzir a desigualdade rural e atuar como uma política de reconhecimento dos agricultores familiares.

\section{O Pronaf na região Sul}

Como já foi citada, a região Sul recebeu mais recursos do Pronaf do que as outras regiões, com $41 \%$ do montante e, assim, verifica-se na Tabela 2 como foi a evolução do repasse do programa de 1999 a 2006.

Ademais, é possível verificar que a região Sul seguiu a tendência do país, no que diz respeito ao volume de recursos, o qual apresentou crescimento médio anual de 27\%. Em relação aos contratos, houve oscilações no período; de 1999 a 2000 apresentou um crescimento de $9 \%$, de 2000 a 2001 os contratos apresentaram queda de $8 \%$, de 2001 a 2002 houve crescimento de $2 \%$, de 2002 a 2003 houve queda de 3\% e no período de 2003-2006 houve um crescimento médio de 7\% ao ano. Essa diferença nas oscilações dos contratos e do volume de recursos pode ser explicada pelas mudanças nos valores médios dos contratos. Nesse período, o menor valor do contrato médio foi de $\mathrm{R} \$ 2.119,49$ em 1999 , enquanto que o maior 
valor chegou a R\$ 5.177,54 em 2006. Em relação à evolução da participação percentual do volume de recursos da região Sul, apesar de apresentar a maior participação percentual em todo o período, entre os anos de 2002 e 2006, houve uma queda média na participação percentual de $9 \%$ ao ano.

Tabela 2 - Número de contratos e volume de recursos do Pronaf para a região Sul 1999-2006

\begin{tabular}{lccccc}
\hline Ano & \multirow{2}{*}{$\begin{array}{c}\text { Número de } \\
\text { contratos }\end{array}$} & Recursos (R\$) & $\begin{array}{c}\text { Média de } \\
\text { recursos por } \\
\text { contrato (R\$) }\end{array}$ & \multicolumn{2}{c}{$\begin{array}{c}\text { \% da participação no } \\
\text { Brasil }\end{array}$} \\
\hline 1999 & 473.231 & $1.003 .006 .889,67$ & $2.119,49$ & 56,44 & Recursos \\
\hline 2000 & 517.290 & $1.117 .665 .036,88$ & $2.160,61$ & 59,93 & 62,77 \\
2001 & 475.760 & $1.284 .976 .185,67$ & $2.700,89$ & 58,12 & 59,42 \\
2002 & 483.761 & $1.442 .535 .102,31$ & $2.981,92$ & 59,73 & 58,32 \\
2003 & 471.167 & $1.609 .845 .201,40$ & $3.416,72$ & 50,97 & 46,94 \\
2004 & 508.723 & $2.204 .431 .238,39$ & $4.333,26$ & 50,23 & 37,80 \\
2005 & 525.064 & $2.482 .245 .221,10$ & $4.727,51$ & 42,90 & 23,78 \\
2006 & 564.011 & $2.920 .187 .487,19$ & $5.177,54$ & 40,75 & 22,10 \\
\hline
\end{tabular}

Fonte: Elaboração própria a partir dos dados do Anuário estatístico do crédito rural - 2006.

Agora se verificam na Tabela 3 os contratos e o volume de recursos de cada estado da região Sul para o período de 1999-2006.

Tabela 3 - Número e participação percentual de contratos e volume de recursos do Pronaf por regiões brasileiras 1999-2006

\begin{tabular}{ccccccc}
\hline \multirow{2}{*}{ Ano } & \multicolumn{2}{c}{ Paraná } & \multicolumn{2}{c}{ Santa Catarina } & \multicolumn{2}{c}{ Rio Grande do Sul } \\
\cline { 2 - 7 } & Contrat & Recursos $(\mathrm{R} \$)$ & $\begin{array}{c}\text { Contrat } \\
\text { OS }\end{array}$ & Recursos $(\mathrm{R} \$)$ & $\begin{array}{c}\text { Contrat } \\
\text { os }\end{array}$ & Recursos $(\mathrm{R} \$)$ \\
\hline \multirow{2}{*}{1999} & 111.918 & $266.737 .234,32$ & 110.084 & $263.900 .350,22$ & 251.229 & $472.369 .305,13$ \\
& $(14 \%)$ & $(15 \%)$ & $(14 \%)$ & $(15 \%)$ & $(32 \%)$ & $(27 \%)$ \\
2000 & 121.279 & $300.515 .658,64$ & 122.987 & $291.461 .431,30$ & 273.024 & $525.687 .946,94$ \\
& $(15 \%)$ & $(16 \%)$ & $(15 \%)$ & $(16 \%)$ & $(33 \%)$ & $(28 \%)$ \\
2001 & 117.758 & $392.910 .140,10$ & 107.188 & $326.345 .749,41$ & 250.814 & $565.720 .296,16$ \\
& $(15 \%)$ & $(18 \%)$ & $(13 \%)$ & $(15 \%)$ & $(31 \%)$ & $(26 \%)$ \\
2002 & 111.681 & $342.102 .560,49$ & 104.302 & $329.751 .696,44$ & 267.778 & $770.680 .845,38$ \\
& $(13 \%)$ & $(14 \%)$ & $(13 \%)$ & $(14 \%)$ & $(32 \%)$ & $(32 \%)$ \\
2003 & 113.070 & $401.364 .313,69$ & 98.518 & $386.067 .732,17$ & 259.579 & $822.413 .155,54$ \\
& $(11 \%)$ & $(13 \%)$ & $(10 \%)$ & $(12 \%)$ & $(26 \%)$ & $(26 \%)$ \\
2004 & 133.182 & $646.492 .061,74$ & 103.551 & $519.126 .317,05$ & 271.990 & $1.038 .812 .859,60$ \\
& $(10 \%)$ & $(15 \%)$ & $(8 \%)$ & $(12 \%)$ & $(20 \%)$ & $(24 \%)$ \\
2005 & 134.346 & $693.409 .315,56$ & 102.654 & $632.636 .849,22$ & 288.064 & $1.156 .199 .056,32$ \\
& $(6 \%)$ & $(12 \%)$ & $(5 \%)$ & $(11 \%)$ & $(13 \%)$ & $(20 \%)$ \\
2006 & 143.457 & $777.692 .517,57$ & 116.674 & $750.950 .086,63$ & 303.880 & $1.391 .544 .882,99$ \\
& $(6 \%)$ & $(11 \%)$ & $(5 \%)$ & $(10 \%)$ & $(12 \%)$ & $(19 \%)$ \\
\hline
\end{tabular}

Fonte: Elaboração própria a partir dos dados do Anuário estatístico do crédito rural - 2006. 
A Tabela 3 mostra que o estado que se destaca na região Sul é o Rio Grande do Sul, pois apresenta em todos os anos do período o maior número de contratos, o maior volume de recursos e o maior percentual na participação de recursos do Pronaf total. O estado de Santa Catarina apresentou em todos os anos do período o menor repasse de recursos da região Sul, mas com valores próximos ao do Paraná. No cenário nacional, os três estados apresentaram um dos maiores números de contratos e volume de recursos repassados pelo Pronaf, com destaque para o estado do Rio Grande do Sul, que apresentou o maior volume de recursos em todo o período.

\section{Metodologia e base de dados}

Esta seção apresenta a estratégia empírica da pesquisa. A metodologia está dividida em seis partes: análise exploratória de dados espaciais, matrizes de pesos espaciais, estatística I de Moran, diagrama de dispersão de Moran, indicadores locais de associação espacial (LISA - Local Indicators of Spatial Association) e fontes de dados e descrição das variáveis.

\subsection{Análise exploratória de dados espaciais (AEDE)}

A análise exploratória de dados espaciais é a técnica utilizada para descrever e visualizar distribuições espaciais, identificar localidades atípicas (outliers espaciais), descobrir padrões de associação espacial (clusters espaciais) e sugerir diferentes regimes espaciais e outras formas de instabilidade (ANSELIN, 1999). O primeiro passo no estudo da AEDE é testar se os dados espaciais estão distribuídos aleatoriamente. Intuitivamente, aleatoriedade espacial significa que os valores de um atributo numa região não dependem dos valores desse atributo nas regiões vizinhas (ALMEIDA, 2012).

A partir da Análise Exploratória de Dados Espaciais é possível extrair medidas de autocorrelação espacial global e local, investigando a influência dos efeitos espaciais por intermédio de métodos quantitativos (ANSELIN, 1988). Contudo, antes de desenvolver a AEDE, é necessário, previamente, estabelecer um arranjo que permita estimar coeficientes que indicam o grau de interação entre as unidades espaciais. 


\subsection{Matrizes de pesos espaciais}

A dependência espacial é uma das características dos dados espaciais e pode se manifestar em diversos campos de estudo. Um aspecto fundamental na determinação da autocorrelação espacial é a consideração do grau de vizinhança no qual se deseja realizar a análise de dependência espacial. Uma vez adotado o critério de vizinhança, pode ser construída a matriz de pesos espaciais (GOLGHER, 2015).

As matrizes de pesos espaciais são baseadas na contiguidade e podem ser definidas de acordo com a vizinhança. Para determinar o grau de conexão da matriz de peso espacial, pode ser utilizado como critério o espaço geográfico, que nesse caso, apoia-se na ideia de proximidade definida de acordo com a contiguidade e/ou com a distância geográfica (ALMEIDA, 2012). Na literatura, as matrizes de pesos espaciais mais utilizadas são as matrizes Rainha, Torre e k vizinhos.

A convenção de contiguidade rainha tem como vizinhos oito regiões, localizadas ao norte, sul, leste, oeste e nas quatro diagonais. Já a matriz torre tem como vizinhos apenas as células destacadas em cor escura, sendo assim, apenas quatro regiões seriam consideradas vizinhas de "B", que são as regiões que estão apenas ao norte, sul, leste e oeste. Na literatura, outra matriz também utilizada é a de $\mathrm{k}$ vizinhos mais próximo, em que considera as regiões mais próximas, e o número de regiões depende do que está sendo pesquisado (ANSELIN, 1995).

A matriz dos $\mathrm{k}$ vizinhos mais próximos consiste em uma matriz binária, no qual sua convenção é determinada pela distância geográfica, que pode ser medida tanto em milhas ou quilômetros.

Formalmente a matriz dos k vizinhos mais próximos é dada por:

$$
\begin{array}{lll} 
& 1 & \text { se } d_{i j} \leq d_{i}(k) \\
w_{i j}(k) & \text { o } & \text { se } d_{i j}>d_{i}(k)
\end{array}
$$

Uma vantagem dessa convenção é a capacidade de manter a conexão da matriz, pois todas as unidades espaciais, independentemente da fronteira, apresentarão o mesmo número de vizinhos. 


\subsection{Autocorrelação espacial global bivariada}

Para testar se os dados estão autocorrelacionados no espaço, pode ser utilizado o método estatístico $I$ de Moran que é um coeficiente de autocorrelação espacial. No estudo da análise exploratória dos dados espaciais (AEDE), é possível obter um coeficiente de autocorrelação espacial global num contexto bivariado. Segundo Almeida (2012), a ideia intuitiva é descobrir se o valor de um atributo observado numa dada região está relacionado espacialmente com os valores de outra variável observada em regiões vizinhas. Assim, é possível calcular a estatística $I$ de Moran para duas variáveis diferentes padronizadas, ou seja, $Z_{1}$ e $Z_{2}$, nesse caso, obtém-se a equação 1 :

$$
\mathrm{I}^{\mathrm{Z}_{1} \mathrm{Z}_{2}}=\frac{\mathrm{Z}_{1}{ }_{1} \mathrm{WZ}_{2}}{\mathrm{Z}_{2}{ }_{2} \mathrm{WZ}_{1}}
$$

Em que $w z_{2}$ é a defasagem espacial da variável $z_{2}$, a matriz $w$ é normalizada na linha. De acordo com Anselin et al. (2003), essa estatística fornece o grau de associação linear (positiva ou negativa) entre o valor para uma variável em uma dada locação $i$ e a média de uma outra variável nas locações vizinhas $j$.

O coeficiente $I$ de Moran fornece três informações: a significância estatística informa sobre os dados estarem distribuídos aleatoriamente ou não, o sinal positivo da estatística $I$ de Moran, desde que significativo, indica que os dados estão concentrados com valores similares nas regiões, o sinal negativo, por sua vez, indica que os dados estão concentrados nas regiões com valores dissimilares; e a magnitude da estatística fornece a força da autocorrelação espacial, quanto mais próximo de 1 ou de -1 mais forte é a autocorrelação, e quanto mais próximo de zero mais disperso estão os dados (ALMEIDA, 2012).

\subsection{Autocorrelação espacial local bivariada}

A análise espacial global pode distorcer os resultados a nível local, embora o resultado geral analisado possa ser relevante sobre uma perspectiva estatística, este esconde algumas particularidades presentes em determinadas localizações do conjunto geográfico. Desse modo, é mais adequado complementar as análises verificando a autocorrelação espacial local (SABATER; TUR; AZORÍN, 2011). A investigação em âmbito local pode ser realizada utilizando-se os indicadores de associação espacial (LISA). Conforme Anselin (1995), a estatística LISA mostra o grau de autocorrelação espacial local, porém, para que isso ocorra, é necessário 
que essa estatística satisfaça a dois critérios: a) esses indicadores devem possuir, para cada observação, uma indicação de clusters espaciais significantes com valores similares ao redor de cada observação; b) o somatório dos indicadores LISA, em todas as regiões, deve ser proporcional ao indicador de autocorrelação espacial global. Segundo Almeida (2012), o indicador de autocorrelação espacial local bivariado (LISA) pode ser obtido por intermédio da equação 2:

$$
\mathrm{I}_{\mathrm{i}}^{\mathrm{Z}_{1} \mathrm{Z}_{2}}=\mathrm{z}_{1 \mathrm{i}} \mathrm{WZ}_{2 \mathrm{i}}
$$

Em que $w_{2}$ é a defasagem espacial da variável padronizada $z_{2 i}$. Nesse caso, somente os vizinhos da observação $i$, definidos conforme a matriz de pesos espaciais, é incluída no cálculo. O indicador local de associação espacial bivariado fornece o grau de associação linear (positiva ou negativa) entre o valor de uma variável em uma determinada região e a média de outra observação nas unidades vizinhas (ANSELIN et al., 2003).

De acordo com Anselin (1995), a estatística LISA é usada para testar a ausência de associação espacial local, assim, deve-se observar se o valor da variável de interesse está dentro ou fora da região crítica definida. Desse modo, os resultados estatísticos do teste superior em magnitude à esperança matemática do I de Moran indicam clusters estatisticamente significativos.

De acordo com Almeida (2012), para cada observação é calculado um coeficiente $\mathrm{I}_{\mathrm{i}}$ com seus respectivos níveis de significância gerando uma elevada quantidade de informações que podem confundir o pesquisador se apresentada em tabela, sendo assim, o autor sugere apresentar esse conjunto de estatísticas no mapa de significância LISA. Ao combinar as informações do diagrama de Moran bivariado com o mapa LISA de significância, é possível obter o mapa de cluster com quatro categorias de regimes espaciais, o que permite uma visualização geográfica mais adequada do grau de concentração das variáveis estudadas.

\subsection{Descrição dos dados}

O estudo contempla os 1188 municípios da região Sul do Brasil, e o período corresponde ao ano de 2006, que apresenta os dados mais atualizados disponíveis para os agricultores familiares. As variáveis utilizadas foram: Agricultores Familiares, que se referem ao percentual de agricultores familiares de cada município da região Sul do Brasil; e o Financiamento do Programa Nacional de 
Fortalecimento da Agricultura Familiar (Pronaf) referente ao valor repassado pelo programa per capita por município da região Sul. Os dados foram extraídos do Ministério do Desenvolvimento Agrário (MDA). As estimativas foram realizadas com o software Geoda. Segue na Tabela 4 a representação das siglas das variáveis utilizadas na análise.

Tabela 4 - Variáveis utilizadas na análise

\begin{tabular}{lc}
\hline Variável & Siglas \\
\hline Agricultores familiares & AGR_FAM \\
Financiamento do Programa Nacional de Fortalecimento da Agricultura & PRON \\
Familiar - Pronaf & \\
\hline
\end{tabular}

Fonte: Elaboração própria.

\section{Resultados}

Nesse item, foi realizada a análise exploratória de dados espaciais das variáveis percentual de agricultores familiares e o valor do financiamento do Pronaf per capita para os municípios da região Sul.

\subsection{Autocorrelação espacial global bivariada}

Na Tabela 5, é possível observar o resultado para autocorrelação espacial entre o percentual de agricultores familiares e o valor do financiamento do Pronaf per capita no ano de 2006. Foram utilizadas seis simulações de matrizes de pesos espaciais, com as seguintes convenções: Rainha, Torre, $\mathrm{k} 2$ vizinhos, $\mathrm{k} 5$ vizinhos, k 10 vizinhos e k 12 vizinhos. Como o valor I de Moran ficou acima do valor esperado $^{\dagger} \mathrm{E}(\mathrm{I})=[-1 /(1188-1)=-0,00084]$ para todas as convenções no ano analisado, pode-se inferir que existe autocorrelação espacial positiva entre o percentual de agricultores familiares e o valor do financiamento do Pronaf per capita municipal a um nível de significância de 1\%. Para a análise, foi utilizada a matriz de pesos espaciais do tipo Torre, pois apresentou o maior valor para o I de Moran.

\footnotetext{
${ }^{\dagger} \mathrm{A}$ esperança do I de Moran é dada por: [-1/ (n-1)], em que n é o número de municípios.
} 


\section{Tabela 5 - Autocorrelação global bivariada (agr_fam vs pron) da região Sul para 0 ano de 2010}

\begin{tabular}{lc}
\hline Matriz de Pesos Espaciais & I de Moran \\
\hline RAINHA & $0,1783^{*}$ \\
TORRE & $0,1799^{*}$ \\
K 2 VIZINHOS & $0,1014^{*}$ \\
K 5 VIZINHOS & $0,1471^{*}$ \\
K 10 VIZINHOS & $0,1515^{*}$ \\
K 12 VIZINHOS & $0,1428^{*}$ \\
\hline
\end{tabular}

Fonte: Elaboração própria a partir dos dados do MDA. Nota: *Nível de significância de 1\%.

O sinal positivo da estatística I de Moran indica que os municípios com elevado percentual de agricultores familiares estão cercados por municípios com alto valor do financiamento do Pronaf per capita, e as localidades com o percentual de agricultores familiares baixo são circunvizinhados por unidades municipais com baixo valor do financiamento do Pronaf por pessoa. Os resultados corroboram com os estudos de Mattei (2005) e Mattei (2007), em que mostram uma concentração no volume de recursos do Pronaf em regiões que apresentam os principais grupos de agricultores familiares. O estudo de Pereira e Nascimento (2014) também mostrou a importância do Pronaf na produção dos agricultores familiares nos municípios do Tocantins. Em decorrência do valor positivo da estatística I de Moran, os dados tendem a se agruparem mais no primeiro quadrante (alto-alto) e no terceiro quadrante (baixo-baixo).

O diagrama de dispersão de Moran bivariado exibe no eixo horizontal o percentual de agricultores familiares e no eixo vertical o valor do financiamento do Pronaf per capita. O valor positivo do coeficiente I de Moran é reforçado pela inclinação positiva da reta de regressão (Figura 1).

A Figura 1 mostra que, no diagrama de dispersão, 27\% dos municípios se concentram no primeiro quadrante, ou seja, aglomeração espacial alto-alto (AA), também $27 \%$ das unidades espaciais estão no segundo quadrante, ou seja, baixoalto (BA), 36\% das unidades municipais estão situadas no terceiro quadrante, sendo assim, consideradas baixo-baixo (BB) e $10 \%$ se concentram no quarto quadrante, caracterizados como regime espacial alto-baixo $(\mathrm{AB})$. 


\section{Figura 1 - Diagrama de Moran bivariado (agr_fam vs pron) para a região Sul no ano de 2006}

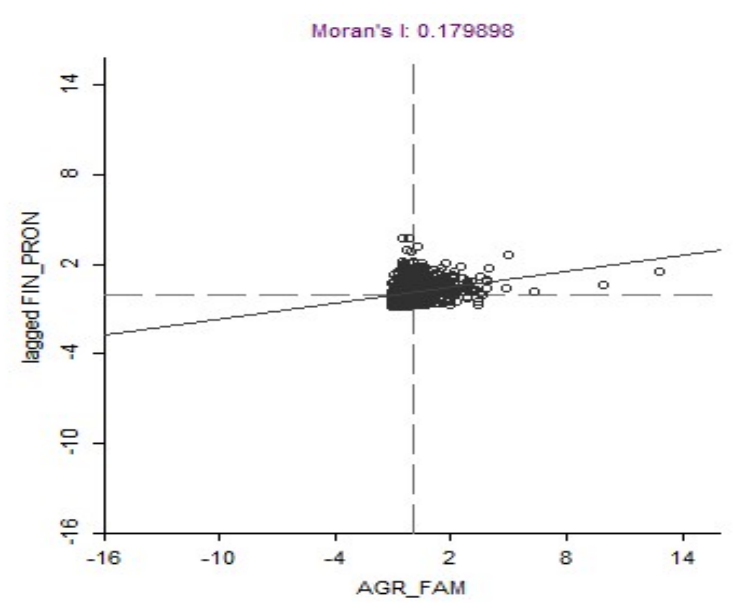

Fonte: Elaboração própria utilizando o software Geoda e os dados do MDA.

Portanto, isso indica as aglomerações da relação entre o número de agricultores familiares e o financiamento do Pronaf. As aglomerações AA indicam municípios que apresentam número de agricultores familiares acima da média e com financiamento do Pronaf acima da média. As concentrações BA apontam municípios com número de agricultores familiares abaixo da média e com o financiamento do Pronaf acima da média. As aglomerações BB indicam municípios que apresentam número de agricultores familiares abaixo da média e com financiamento do Pronaf abaixo da média. As concentrações $A B$ apontam municípios com número de agricultores familiares acima da média e com o financiamento do Pronaf abaixo da média. Desse modo, para verificar os clusters espaciais estatisticamente significativos, foi realizada a análise da autocorrelação espacial bivariada.

\subsection{Autocorrelação espacial local bivariada}

O mapa de cluster do AGR_FAM vs PRON, apresentado na Figura 2, permite verificar onde foram formados os agrupamentos espaciais estatisticamente significativos a $5 \%$, divididos em quatro categorias de associação espacial. As localidades destacadas em vermelho representam os clusters espaciais alto-alto (AA), as unidades denotadas em azul no mapa exibem os regimes espaciais baixo- 
baixo (BB), e em vermelho claro e azul claro estão os clusters atípicos alto-baixo $(\mathrm{AB})$ e as unidades municipais baixo-alto (BA), respectivamente.

Conforme mostra o mapa de cluster que relaciona a quantidade de agricultores familiares e o financiamento do Pronaf, observa-se que 32\% (382) dos municípios foram estatisticamente significativos a $5 \%$. É possível verificar também que a formação de cluster do tipo alto-alto (AA) ficou concentrada principalmente no estado do Rio Grande do Sul. Em torno de 96 municípios compõem o agrupamento com alto percentual de agricultores familiares e elevado valor do financiamento do Pronaf per capita na região Sul. Dentre os municípios estatisticamente significativos, foi verificado no agrupamento espacial AA que o Rio Grande do Sul possui 64\% (61) dos seus municípios. Santa Catarina apresentou $8 \%$ (8) dos municípios e o Paraná 28\% (27). Sendo que no Paraná, as maiores concentrações foram nas mesorregiões Sudeste, Centro-Sul e Sudoeste; em Santa Catarina, a maior concentração ficou situada na mesorregião Oeste; e no Rio Grande do Sul, as maiores concentrações ficaram nas regiões Centro-Oriental e Sudeste.

Por outro lado, no estado do Paraná foi verificado a maior concentração de agrupamentos espaciais do tipo baixo-baixo (BB), nos quais aparecem 170 municípios que contemplam baixo percentual de agricultores familiares e baixo valor do financiamento do Pronaf per capita na região Sul. Ao verificar os estados com unidades nesse cluster, foi observado que dos municípios significativos, o Paraná apresentou 56\% (95) dos municípios, Santa Catarina apresentou 22\% (37) e o estado do Rio Grande do Sul apresentou 22\% (38) dos municípios. Visto que no Paraná, as mesorregiões do Norte, Noroeste e Metropolitana de Curitiba apresentaram as maiores concentrações de clusters baixo-baixo (BB); em Santa Catarina a maior concentração desses clusters foi nas mesorregiões do Vale do Itajaí, Norte e na Grande Florianópolis; e no Rio Grande do Sul as concentrações se situaram nas mesorregiões Sudoeste e Metropolitana de Porto Alegre. 
Figura 2 - Mapa de cluster bivariado (agr_fam vs pron) para o ano de 2006

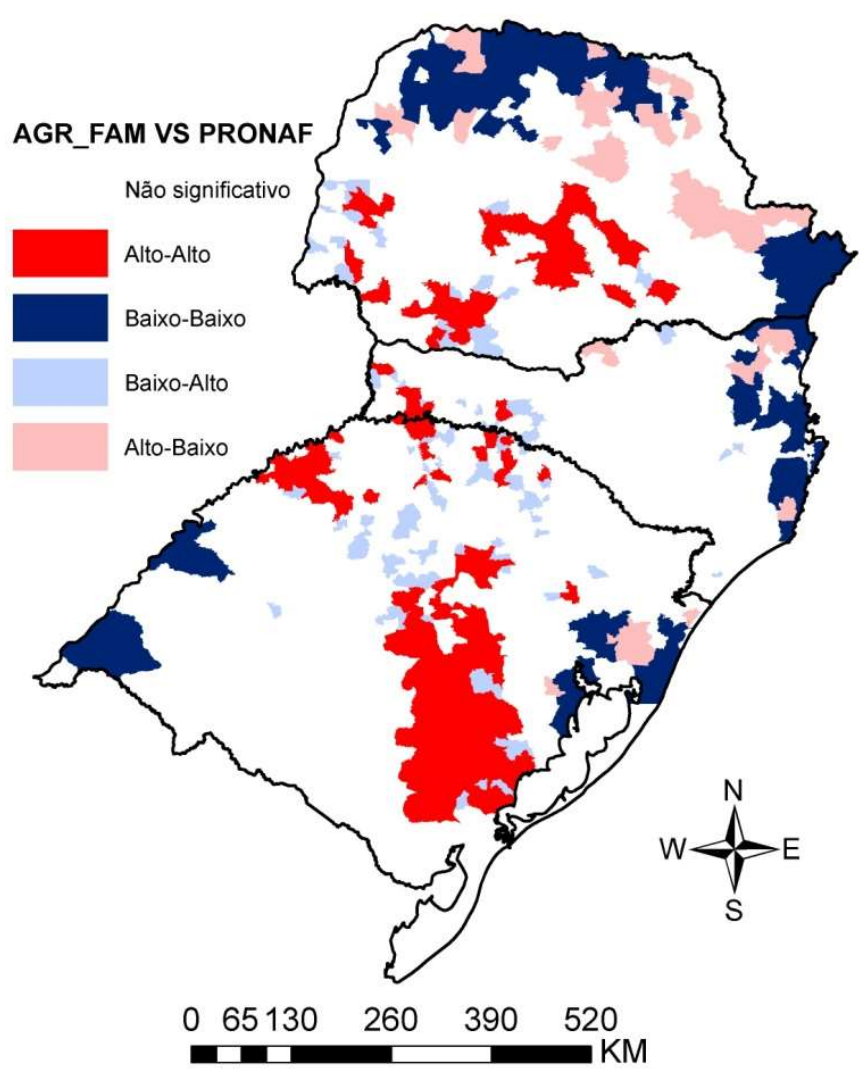

Fonte: Elaboração própria utilizando o software Quantum GIS e os dados do MDA.

Portanto, foi identificada a autocorrelação espacial positiva entre o financiamento do Pronaf e os agricultores familiares, com as principais aglomerações de alto número de agricultores e alto financiamento do Pronaf no Rio Grande do Sul. Isso indica que o programa é de grande importância para atender seu principal objetivo, que é reduzir a pobreza no meio rural, porém, ainda não é totalmente suficiente.

\section{Considerações finais}

O presente estudo teve o intuito de identificar a relação espacial entre o percentual de agricultores familiares e o valor do financiamento do Pronaf per capita para os municípios do Sul do Brasil no ano de 2006, o qual procurou verificar se municípios com elevado percentual de agricultores familiares são cercados por municípios que recebem um alto financiamento do Pronaf per capita. 
A análise global mostrou que o resultado do $I$ de Moran foi de 0,1799, o que aponta uma autocorrelação espacial positiva entre as variáveis. Isso indica que os municípios com alto (baixo) percentual de agricultores familiares estão circunvizinhados por municípios com elevado (baixo) financiamento do Pronaf per capita.

O resultado da análise espacial local revelou que o estado do Rio Grande do Sul apresentou a maior concentração de cluster do tipo alto-alto (AA), sendo que, dentro do estado, as maiores concentrações ficaram nas mesorregiões CentroOriental e Sudeste. Por outro lado, os estados do Paraná e Santa Catarina apresentaram as maiores concentrações de clusters do tipo baixo-baixo (BB), sendo que, no estado do Paraná, as maiores concentrações ficaram nas mesorregiões do Norte, Noroeste e Metropolitana de Curitiba; e no estado de Santa Catarina, as maiores concentrações se situaram nas mesorregiões do Vale do Itajaí, Norte e na Grande Florianópolis. Portanto, pode-se concluir que há focalização do Pronaf em regiões que mais concentram agricultores familiares no Sul do país.

\section{Referências}

ALMEIDA, E. Econometria espacial aplicada. Alínea: Campinas: SP, 2012.

ANJOS, F. S.; GODOY, W. I.; CALDAS, N. V.; GOMES, M. C. Agricultura Familiar e Políticas Públicas: o Impacto do Pronaf no Rio Grande do Sul. Revista de Economia e Sociologia Rural, v. 42, n. 03, p. 529-548, 2004.

ANSELIN, L. Spatial econometrics: methods and models. Boston: Klumer academic, 1988.

ANSELIN, L. SYABRI, I.; SMIRNOV, O. Visualizing Multivariate Spatial Correlation with Dynamically Linked Windows. University of Illinois, 2003.

ANSELIN, L. Local indicators of spatial association - (LISA). Geographical Analysis, v. 27, n. 2, p. 93-115, 1995.

ANSELIN, L. Interactive techniques and exploratory spatial data analysis. In: LONGLEY, M. F.; GOODCHILD, D. J.; MAGUIRE, RHIND, D. W. Geographical information systems: principles, techniques, management and Applications. New York: Jonh Wiley. p. 251-264, 1999. 
BACEN - Banco Central do Brasil. Anuário estatístico do Crédito Rural 2006.

Disponível em: <https://www.bcb.gov.br/estabilidadefinanceira/anuario_estat _credrural $>$. Acesso em: 14 out. 2016.

BATISTA, H. R.; NEDER, H. D. Efeitos do Pronaf Sobre a Pobreza Rural no Brasil (2001-2009). Revista de Economia e Sociologia Rural. Piracicaba, v. 52, s. 1, p. S147-S166, 2004.

BIANCHINI, V. Vinte anos do Pronaf, 1995-2015: avanços e desafios. Brasília: SAF/MDA, 2015.

BRASIL. IBGE. Censo Agropecuário, 2006. Disponível em: <ftp://ftp.ibge.gov.br /Censos/Censo_Agropecuario_2006>. Acesso em: 14 out. 2016.

GOLGHER, A. B. Introdução à econometria espacial. Paco Editorial: Jundiaí: SP, 2015.

MATTEI, L. Impactos do Pronaf: análise de indicadores. Núcleo de Estudos Agrários e Desenvolvimento Rural: Brasília: DF, 2005.

MATTEI, L. Políticas de Apoio ao Desenvolvimento da Agricultura Familiar no Brasil: O Caso Recente do Pronaf. Revista Econômica do Nordeste. Fortaleza, v. 38, n. 1, 2007.

MATTEI, L. Programa Nacional de Fortalecimento da Agricultura Familiar (PRONAF): concepção, abrangência e limites observados. In. Anais. IV Encontro da Sociedade Brasileira de Sistemas de Produção - SBSP. Belém, 2001.

MINISTÉRIO DO DESENVOLVIMENTO AGRÁRIO. Agricultores Familiares. Brasília. 2016. Disponível em: <http:/www.mda.gov.br/>. Acesso em: 15 out. 2016.

MINISTÉRIO DO DESENVOLVIMENTO AGRÁRIO. Agricultores Familiares. Base de dados do Crédito Pronaf: Anuário estatístico do crédito rural 2006. Disponível em: <http://www.mda.gov.br/>. Acesso em: 15 de outubro de 2016.

MICHELLON, E.; PELIZER, T. O financiamento para a aquisição de insumos nas revendas do Paraná no século XXI. In: Anais. XLIV Congresso da Sociedade Brasileira de Economia e Sociologia Rural - Sober. Fortaleza, 2006.

MICHELlON, E.; KRETZMANN, C. K.; GONÇALVES, D. F. Análise Conjuntural do Crédito Rural: Agricultura Familiar versus Agricultura Patronal. In. Anais. XLV Congresso da Sociedade Brasileira de Economia, Administração e Sociologia Rural - Sober. Londrina, 2007. 
PEREIRA, E. L.; NASCIMENTO, J. S. Efeitos do Pronaf sobre a Produção Agrícola Familiar dos Municípios Tocantinenses. Revista de Economia e Sociologia Rural, v. 52, n. 1, p. 139-156, 2014.

RESENDE, C. M.; MAFRA, R. L. M. Desenvolvimento Rural e Reconhecimento: tensões e dilemas envolvendo o Pronaf. Revista de Economia e Sociologia Rural, v. 54, n. 2, p. 261-280, 2016.

SABATER, L. A.; TUR, A. A.; AZORÍN, J. M. N. Análise Exploratória dos Dados Espaciais. In: COSTA, J.S.; DENTINHO, T.P.; NIJKAMP, P. Compêndio de Economia Regional: métodos e técnicas de análise regional. v. 2. Principia, p. 259291, 2011. 